Physica B 165\&166 (1990) 95-96

North-Holland

\title{
DESIGN AND CONSTRUCTION OF A 19-CHANNEL DC-SQUID NEURONAGNETONETER
}

\author{
H. J.M. TER BRAKE, J. FLOKSTRA, E.P. HOUWMAN, D. VELDHUIS, W. JASZCZUK, A. MARTINEZ* \\ and H. ROGALLA \\ University of Twente, Faculty of Applied Physics, Low Temperature Group, \\ P.O.Box 217, 7500 AE Enschede, The Netherlands. \\ University of Zaragoza, Dept. of Electrical Engineering and Informatics, Maria Zambrano 50 , \\ 50015 Zaragoza, Spain.
}

\begin{abstract}
The 19-channel DC-SQUID neuromagnetometer which is under construction at the University of Twente is described. Several aspects of this development are considered: the DC-SQUID sensor, the design of the SQUID module, the arrangement of the 19 gradiometers, and the electronics, including the output transformer, the preamplifier and the control and detection section. The completed system will be installed in our magnetically shielded room.
\end{abstract}

\section{INTRODUCTION}

During the last few years biomagnetic instrumentation has changed from single-channel SQUID systems to multichannel magnetometer units (1). In this way the magnetic field distribution around a subject's body can be measured much faster and more rellable. Furthermore, spontaneous activity in the body can now be studied. Multichannel systems of about 20 to 30 channels are developed by or are under construction in university groups as for instance in Helsinki and Rome and in industries like BTi, Siemens and Philips.

At the University of Twente a 19 channel DCSQUID magnetometer for brain research is under construction. In this paper several aspects of the system are shortly described. These concern the sensing coils, which have been optimized with respect to the signal-to-noise ratio of the system, the cross-talk elimination by means of external feedback, electronic noise cancellation, the DC-SQUID sensor and the design of the SQUID module, and finally the electronics including the output transformer, the preamplifier and the control and detection section.

\section{SENSOR ASSEMBLY}

The gradiometers are optimized with respect to the signal-to-noise ratio according to the method presented in ref. 2 . The resulting design, based on a system-noise level of $(2-6) \cdot 10^{-6} \phi_{0} / \sqrt{H z}$ and an input inductance of $(0.2-0.4) \mu \mathrm{H}$ is: diameter $20 \mathrm{~mm}$, baseline $40 \mathrm{~mm}$, 2 times 3 turns separated by $0.5 \mathrm{~mm}$ and $6 \mathrm{~mm}$ for the proximal and the compensating coil section, respectively. The gradiometers are distributed in a hexagonal configuration over the bottom of a CTF-SST 140 cryostat having a curvature with a radius of $125 \mathrm{~mm}$. The sensing-coil holders and the support are presented in fig. 1. The material used is tufnol, a textile/epoxy composite.
Because the calculated cross-talk between two neighbouring channels is about $5 \%$ we apply external feedback. The output of each SQUID system is in this case fed back to the corresponding flux-transformer circuit (3). Our shielded room (having only one $\mu$-metal wall) suppresses lowfrequency fields by a factor of about 10 . As a further reduction, electronic noise cancellation can be applied (4). For this purpose three orthogonal $1-\mathrm{cm}$ loops, connected to SQUID systems, are installed as references for the ambient magnetic noise. A first-order gradiometer may be incorporated as an additional noise reference. All in all the magnetometer therefore comprises 22 to 23 SQUID systems.

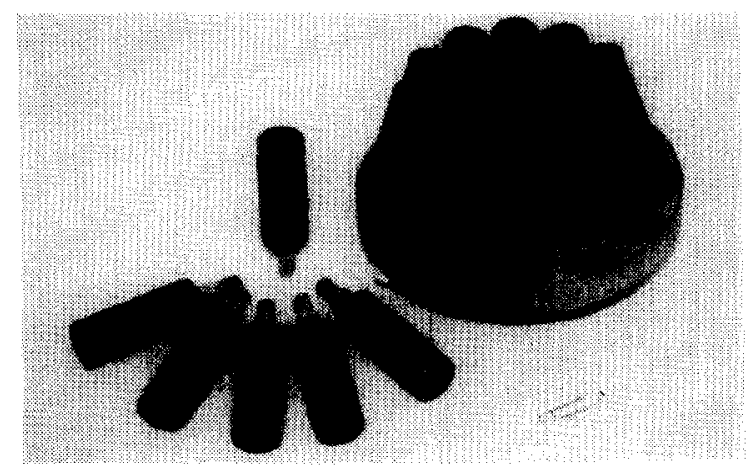

fig. 1: The sensing-coil holders and the support, partly assembled.

The DC-SQUIDs are fabricated based on the $\mathrm{Nb} / \mathrm{Al}, \mathrm{AlO} \mathrm{x}_{\mathrm{x}} / \mathrm{Nb}$ technology. The junctions are of high quality with a gap voltage of $2.9 \mathrm{mV}$, an IcRN product that is $80 \%$ of the theoretical value and a high subgap resistance leading to $V_{m}=70 \mathrm{mV}$ at $4.2 \mathrm{~K}$. The junctions are shunted by 
a Pd resistor of about $3 \Omega$ and are part of a standard washer type SQUID configuration. DCSQUIDs with different inductances, varying from 110 to $240 \mathrm{pH}$, have been fabricated. The planar input coil consists of 30 to 42 turns corresponding to an input inductance of $0.2-0.4 \mu \mathrm{H}$. Apart from this standard type SQUID, also resistively and inductively shunted configurations were made, which allow for a larger SQUID inductance. Thus the number of turns of the input coil may be reduced. These different approaches are compared to come to the best SQUID design for our application (5).

Each SQUID is placed on a solid Nb body which fits into a cylindrical superconducting shleld. As depicted in fig.2, this module is divided into compartments to maximize the shielding of the different sections.

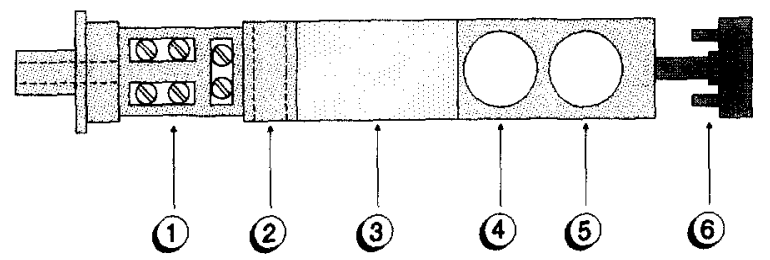

fig. 2: Compartments of the SQUID module

1: screw connections for sensing coil and feedback inductor

2: hole for feedback inductor

3: plane for SQUID chip

4: hole for output transformer

5: hole for capacitor (tuning the transformer)

6: connector

\section{ELECTRONICS}

The preamplifier section is placed directly on top of the cryostat in an aluminium enclosure. It consists of U311 FETs in a cascode configuration, loaded with an RCL-network resonating at the modulation frequency of $100 \mathrm{kHz}$. The quality factor is about 5 and the preamplifier gain about 50. In order to reduce the effect of the preamplifier nolse ( $1 \mathrm{nV} / \sqrt{\mathrm{Hz}})$, a small transformer is used between the SQUID and the preamplifier. It is tuned to $100 \mathrm{kHz}$ via a capacitive shunt. Without the transformer, the preamplifier would give an equivalent noise in the SQUID of about $2.10^{-5} \phi_{0} / \sqrt{H z}$, the forward transfer of the SQUID being roughly $70 \mu \mathrm{V} / \phi_{\circ}$ (5). In order to limit the noise contribution to $10^{-6} \phi_{0} / \sqrt{\mathrm{Hz}}$, the tuned transformer should thus have a gain of at least 20. A moderate Q-factor of 10 was chosen to give a bandwidth of $10 \mathrm{kHz}$. To realize an overall gain of more than 20 , the transformer voltage-gain should therefore be larger than 2. A prototype transformer has been made of $0.1 \mathrm{~mm}$ copper wire. It has a length of 5 $\mathrm{mm}$ and an outer diameter of $5.3 \mathrm{~mm}$, thus fitting in the $7-\mathrm{mm}$ hole of the SQUID module. The prototype consisted of 94 primary and 371 secondary turns. The measured inductances are respectively $14.9 \mu \mathrm{H}(20.6 \mu \mathrm{H})$ and $204 \mu \mathrm{H}(297 \mu \mathrm{H})$ whereas the coupling factor appeared to be as high as $0.94(0.97)$ (transformer voltage-gain 3.5 (3.7)). The values between brackets were measured outside the superconducting enclosure.

The control and detection electronics is realized in a miniaturized version on a single Eurocard for each channel. Because of the miniaturization and the fact that the system is not designed to be a general-purpose magnetometer, several parameters have been fixed among which the sensitivity and the frequency bandwidth $(5 \mathrm{kHz})$. The sensitivity is $1 \mathrm{~V}$ at the output for $1 \phi_{0}$ in the SQUID. The dynamic range of $10 \mathrm{~V}$ corresponds to an effective fleld of about $3 \mathrm{nT}$ in the gradiometer. The power supplies and the bias-current supplies are independent for each channel, whereas the $100 \mathrm{kHz}$ modulation for all channels is obtained from an external oscillator. The electronics have been developed independently from the DC-SQUID devices. System parameters as dynamic behaviour, the gain of the feedback path, and the open-loop gain have been adjusted by means of a DC-SQUID simulator (6).

Before $A / D$ conversion some analogue filtering is necessary. For this purpose we developed a 50 $\mathrm{Hz}$ adaptive notch filter that suppresses the mains frequency by more than $60 \mathrm{~dB}$ with a bandwidth of only $1 \mathrm{~Hz}$. Further, high-pass and lowpass filters will be applied, the latter also functioning as anti-alias filters for the $1 \mathrm{kHz}$ sampling frequency. Thus signals can be measured without significant phase-shift up to a frequency of $100 \mathrm{~Hz}$, which is adequate for most biomagnetic experiments.

\section{CONCLUSION}

We presented the design of our 19-channel DC-SQUID neuromagnetometer. Various aspects concerning the sensor assembly and the electronics are shortly discussed. After the first test experiments of the completed system, an extensive description will be given elsewhere. The neuromagnetometer will be installed in the magnetically shielded room of the Biomagnetic Center Twente. It will be used among other applications for studies on epilepsy and cognitive processes.

\section{REFERENCES}

(1) see e.g. G.L.Romani, Physica 126 B (1984) 70.

(2) H.J.M.ter Brake, Sensing-coil optimization based on signal-to-noise ratio, to be published in Proceedings Biomagnetism VII, New York 1989.

(3) H.J.M. ter Brake et al., Cryogenics 26 (1986) 667

(4) H.J.M. ter Brake et al., J. Phys. E; Sci. Instrum. 22 (1989) 560

(5) E.P.Houwman et al., Comparison of shunted DC-SQUIDs with large $\beta$, this volume.

(6) A. Martinez et al., A low-nolse multi-step SoUID simulator, to be published in Cryogenics. 